\title{
How does adding and removing liquid from socket bladders affect residual-limb fluid volume?
}

\author{
Joan E. Sanders, PhD; ${ }^{*}$ John C. Cagle, BSE; Daniel S. Harrison, BS; Timothy R. Myers, MME; Kathryn J. \\ Allyn, CPO \\ Department of Bioengineering, University of Washington, Seattle, WA
}

\begin{abstract}
Adding and removing liquid from socket bladders is a means for people with limb loss to accommodate residuallimb volume change. We fit 19 people with transtibial amputation using their regular prosthetic socket with fluid bladders on the inside socket surface to undergo cycles of bladder liquid addition and removal. In each cycle, subjects sat, stood, and walked for $90 \mathrm{~s}$ with bladder liquid added, and then sat, stood, and walked for $90 \mathrm{~s}$ again with the bladder liquid removed. The amount of bladder liquid added was increased in each cycle. We used bioimpedance analysis to measure residual-limb fluid volume. Results showed that the preferred bladder liquid volume was $16.8+/-8.4 \mathrm{~mL}$ (mean $+/$ - standard deviation), corresponding with $1.7 \%+/-0.8 \%$ of the average socket volume between the bioimpedance voltage-sensing electrodes. Residual-limb fluid volume driven out of the residual limb when bladder liquid was added was typically not recovered upon subsequent bladder liquid removal. Of the 19 subjects, 15 experienced a gradual residual-limb fluid volume loss over the test session. Care should be taken when implementing adjustable socket technologies in people with limb loss. Reducing socket volume may accentuate residual-limb fluid volume loss.
\end{abstract}

Key words: accommodation, amputation, bioimpedance, fluid insert, interface pressure, prosthesis, prosthetic socket, residual limb, transtibial, volume.

\section{INTRODUCTION}

There are several methods for people with limb loss to accommodate daily changes in the volume of their residual limb [1]. The most common is to add or remove prosthetic socks. Adding or removing socks is simple and inexpensive to implement, but the method is inconvenient because the person must remove his or her prosthesis and overlying clothing to effect the change. Another method is to periodically doff the prosthesis to allow recovery of some of the residual-limb volume lost earlier in the day. Doffing the prosthesis releases socket pressures on the residual limb and can be effective at facilitating residuallimb volume recovery [2-3]. But, like adding or removing socks, the prosthesis must be doffed, which many people find inconvenient. Further, they cannot ambulate during the doffing period unless they used supporting aides. Another method, inducing a vacuum within the prosthetic socket (termed "vacuum-assist" or "elevated vacuum"), does not require the prosthesis be doffed. By applying vacuum pressure to the socket, vacuum-assist devices are intended to pull residual-limb soft tissues outward during the swing phase of gait or during low weightbearing conditions and help draw fluid into the residual limb, retarding daily fluid volume loss [4]. However, vacuum-assist technology requires a precisely designed socket shape and it can be challenging to maintain the vacuum [5]. A liner or sleeve material tear or defect can

\footnotetext{
Abbreviations: $\mathrm{ABI}=$ ankle-brachial index, $\mathrm{ASGP}=$ ambulatory strain-gage plethysmography, BMI = body mass index, $\mathrm{OBP}=$ orthostatic blood pressure, $\mathrm{RF}=$ radio frequency.

*Address all correspondence to Joan E. Sanders, PhD; University of Washington, 3720 15th Ave NE, Seattle, WA 98195; 206-221-5872; fax: 206-685-3300.
}

Email: jsanders@u.washington.edu

http://dx.doi.org/10.1682/JRRD.2012.06.0121 
cause a loss of vacuum pressure. Another method for accommodation is an adjustable socket that reduces in size during periods of activity to achieve good suspension and stability and enlarges in size during inactivity to facilitate fluid volume recovery into the residuum. An adjustable socket is similar in concept to sock addition or removal and periodic doffing but does not require the prosthesis be removed to effect accommodation. In this sense, adjustable sockets are convenient for the prosthesis user.

Several adjustable socket technologies are available or are emerging. These include air-inflatable inserts (e.g., Pneu-Fit, Little Rock Prosthetics; Little Rock, Arkansas), liquid-filled bladders (e.g., Active Contact System, Simbex; Lebanon, New Hampshire), and magneto-rheological liquid systems [6]. Most air-filled inserts operate effectively over only a narrow volume range, in part because air is compressible [7]. Water-based solutions are essentially incompressible; thus, liquid-filled bladders do not have this limitation. The Simbex Active Contact System uses a passive mechanical control system to adjust the volume of liquid-filled bladders [8-9]. However, it is not often used by practitioners, in part because there is no billing code for the technology. Other inventions extending from the Simbex Active Contact System have been developed [10-11].

Part of the challenge of creating an effective adjustable socket system for people with transtibial limb loss is that changing the bladder liquid volume may affect residual-limb volume. Currently, the interplay between filling or emptying the bladders and changes in residual-limb fluid volume is unknown. The issue is relevant for people with transtibial amputation because socket fit is so sensitive to small volume changes. A study of 10 subjects with transtibial limb loss showed that an experienced practitioner, using static fitting procedures, identified sockets oversized by just $0.25 \mathrm{~mm}$ mean radial thickness (approximately half the thickness of a new 3-ply Soft Sock [KnitRite Inc; Kansas City, Kansas] while worn on a residual limb during walking [12]) as needing sock addition or other modification [13]. If increasing bladder liquid volume reduces residual-limb fluid volume, then more bladder liquid may need to be added, either manually or automatically, to compensate. The residual limb may enter a positive feedback loop: further residual-limb fluid volume loss occurs, requiring further bladder liquid to be added, and the cycle repeated. A period of residual-limb fluid volume recovery or some other treatment may need to be introduced to retard continued fluid volume loss.

We hypothesize that injecting and removing liquid from bladders inside the socket affects residual-limb fluid volume, with the magnitude of the change depending on how much liquid is injected and when it is injected. We expect that residual-limb fluid volume easily reduces (i.e., residual-limb fluid volume lost equals bladder liquid injected) when small amounts of liquid are initially injected into bladders. ("Deformable" in Figure 1(a)). Further, fluid driven out of the residual limb does not quickly return when this small amount of bladder liquid is removed (region 1 in Figure 1(b)). A practitioner assumes this fluid loss when asking a patient to stand (a)

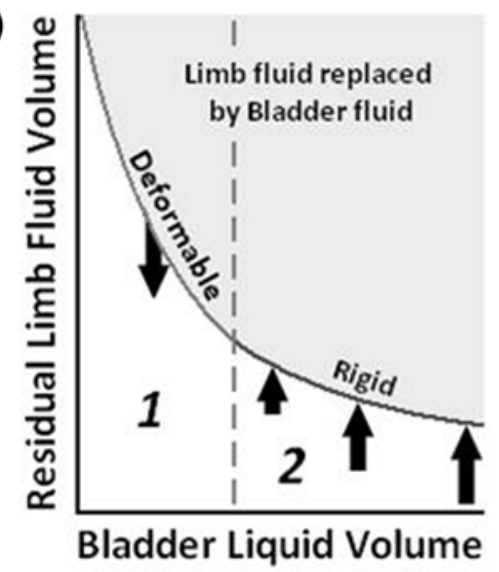

(b)

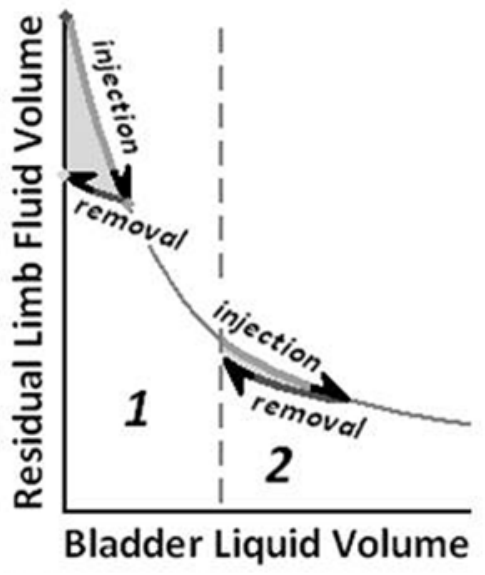

(c)

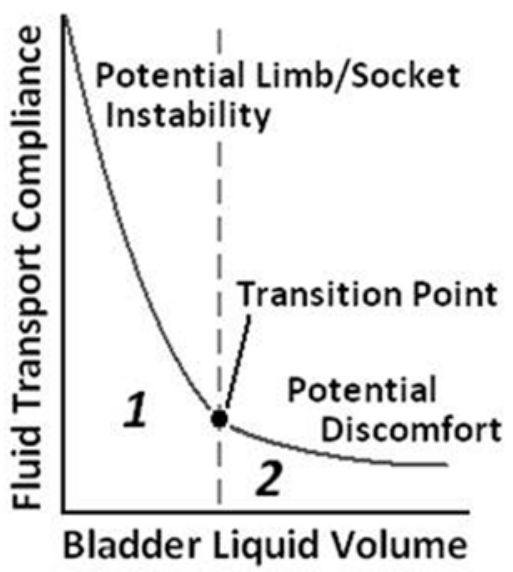

Figure 1.

Plots indicate expected interactions between bladder liquid volume changes and residual-limb fluid volume. (a) Fluid injection response. (b) Fluid injection and removal cycles. (c) Compliance. 
with weight-bearing for several minutes. Physiologically, since socket donning increases interstitial fluid pressure, we would suspect that much of this exited fluid was blood driven out through the venous vasculature. Because interstitial pressure remains elevated as long as the socket remains donned, the arterial to interstitial pressure gradient continues to be reduced compared with its level when the prosthesis was doffed. Thus, we would not expect residual-limb fluid volume recovery, as illustrated in Figure 1(b). Our physiologic hypothesis has not been tested and is thus conjecture at this point. We created the term residual-limb "fluid transport compliance" to characterize the inability of the limb to recover fluid volume after insert liquid is injected and removed (Figure 1(c)). Fluid transport compliance is the difference in slope between the injection slope and the removal slope (labeled in Figure 1(b)). A high compliance means that the residual limb does not "bounce back" to the fluid volume level before the cycle of bladder liquid injection and removal.

When the amount of liquid injected into the bladders is increased, we expect resistance to residual-limb fluid outflow to increase ("Rigid" in Figure 1(a)). After the residual-limb fluid volume loss experienced in region 1, there is less residual-limb fluid available to displace out of the residual limb. As a result, almost all of the fluid driven out of the residual limb returns (region 2 in Figure 1(b)), and compliance decreases (region 2 in Figure 1(c)). We hypothesize that there is a transition point in the compliance curve representing the preferred bladder volume for the prosthesis user. We expect the transition point to be at the intersection between the steep slope and shallow slope portions of the curve because we suspect this point is where mechanical stability is achieved. In other words, the socket is tight enough that limb-socket mechanical coupling is stable, but at the same time, socket pressures are not so excessive that the socket is uncomfortable. More liquid addition would only increase limb-socket stresses and induce discomfort; thus, we would not anticipate that subjects would prefer a higher bladder fluid setting.

In this research, we focused on better understanding how filling and emptying liquid bladders affected residual-limb fluid volume on a group of subjects with transtibial amputation. The intent was to provide a base understanding from which to develop possible strategies to set bladder liquid volumes for individual patients. This insight should facilitate the evaluation of emerging con- trol systems and their enhancement to automatically adjust socket size for prosthesis users to induce a stable residual-limb fluid volume over the day with low risk of injury, instability, or discomfort.

We chose not to use an automated liquid-filled bladder system (e.g., Volume Control System, Simbex) that adjusts liquid volume based on bladder pressure for two primary reasons. First, we would have had no way of measuring how much fluid was actually within the bladders at any given time. Second, we would have introduced uncontrolled variables to the research study, specifically the amount of liquid added and when liquid was added. Investigation of a relationship between bladder liquid volume and residual-limb fluid volume, which we consider scientifically essential toward effective implementation of liquid bladders in prosthetic sockets, would not have been possible. Evaluation of automated liquid-filled bladder systems controlled by pressure sensing compared with volume sensing would be an appropriate next step after the hypotheses posed here are addressed and an understanding of relationships between bladder liquid volume and residual-limb fluid volume is achieved.

In this research, we address the following questions regarding a group of subjects with transtibial limb loss using prosthetic limbs:

1. What range of bladder liquid volume injection was acceptable?

2. Was there a consistent relationship between liquid volume injected and residual-limb fluid volume change?

3. Did residual-limb fluid volume return to pre-bladderinjection levels when bladder liquid was removed?

4. If not, did the limb become noncompliant as more liquid was injected (i.e., fluid out = fluid in for an inject/ remove cycle) and was this point the location the prosthesis user preferred for bladder liquid volume during ambulation?

\section{METHODS}

\section{Subjects}

Volunteers were considered for inclusion in this study if they had a transtibial limb amputation as least 18 mo prior and were using a definitive prosthesis safely and comfortably for at least $5 \mathrm{~h} / \mathrm{d}$. Other inclusion criteria included the capability of treadmill walking at a selfselected walking speed for at least 2 min continuously. The residual limb needed to be at least $9 \mathrm{~cm}$ in length to allow 
adequate distance between voltage-sensing electrodes (see "Sockets and Instrumentation" section). Exclusion criteria included current skin breakdown, inability to wear the prosthesis for at least $1.5 \mathrm{~h}$ continuously, and inability to shift from standing to supine posture within $30 \mathrm{~s}$ (necessary for vascular tests). The bladders were positioned so that the long axes of the bladders were parallel to the residual-limb longitudinal axis. If the practitioner deemed the socket too small to allow all four bladders to be positioned, then one of the bladders was removed. Tubes from the bladders exited at the socket brim. We used Y-connectors to connect the bladder tubes to one common tube. A stopcock was positioned at the end of the common tube to allow a $100 \mathrm{~mL}$ syringe to be connected to add water to the bladders during the study or to close off the system so that no liquid escaped. We did not control the amount of liquid to each individual bladder but instead controlled the liquid within the entire bladder system.

\section{Sockets and Instrumentation}

Participants used their regular prosthesis during the study. We affixed polyurethane bladders of external dimension $12.7 \times 3.5 \mathrm{~cm}$ (liquid-filled region dimension $11.8 \times 2.5 \mathrm{~cm}$ ) and thickness $0.8 \mathrm{~mm}$ when unfilled to the inside socket surface with double-stick tape at the following locations: lateral tibial flare; medial tibial flare; posterior lateral, distal to the popliteal fossa; and posterior medial, distal to the popliteal fossa. The bladders were positioned at a level on the residual-limb longitudinal axis between the voltage-sensing electrodes and in some cases at the level of the proximal voltage-sensing electrode (Figure 2). We selected these positions because they were consistent with Simbex's recommended locations for their Active Contract System. The locations were not expected to restrict blood flow in the residual limb. The bladders were made by radio frequency (RF)welding together two pieces of 15 -gauge polyurethane film at the edge. A $2.5 \mathrm{~mm}$ inner diameter polyurethane tube was RF-welded into place at the same time to provide a channel for liquid to enter and exit the bladder. We inserted and glued a $10.2 \mathrm{~cm}$-long tube made of Tygon into the end of the polyurethane tube such that it was within the bladder. Without this tube, liquid was difficult to remove from the bottom of the bladder when the subject wore the socket.

We used bioimpedance analysis to assess residuallimb fluid volume. Bioimpedance is a technique commonly used for body composition and body fat assess-

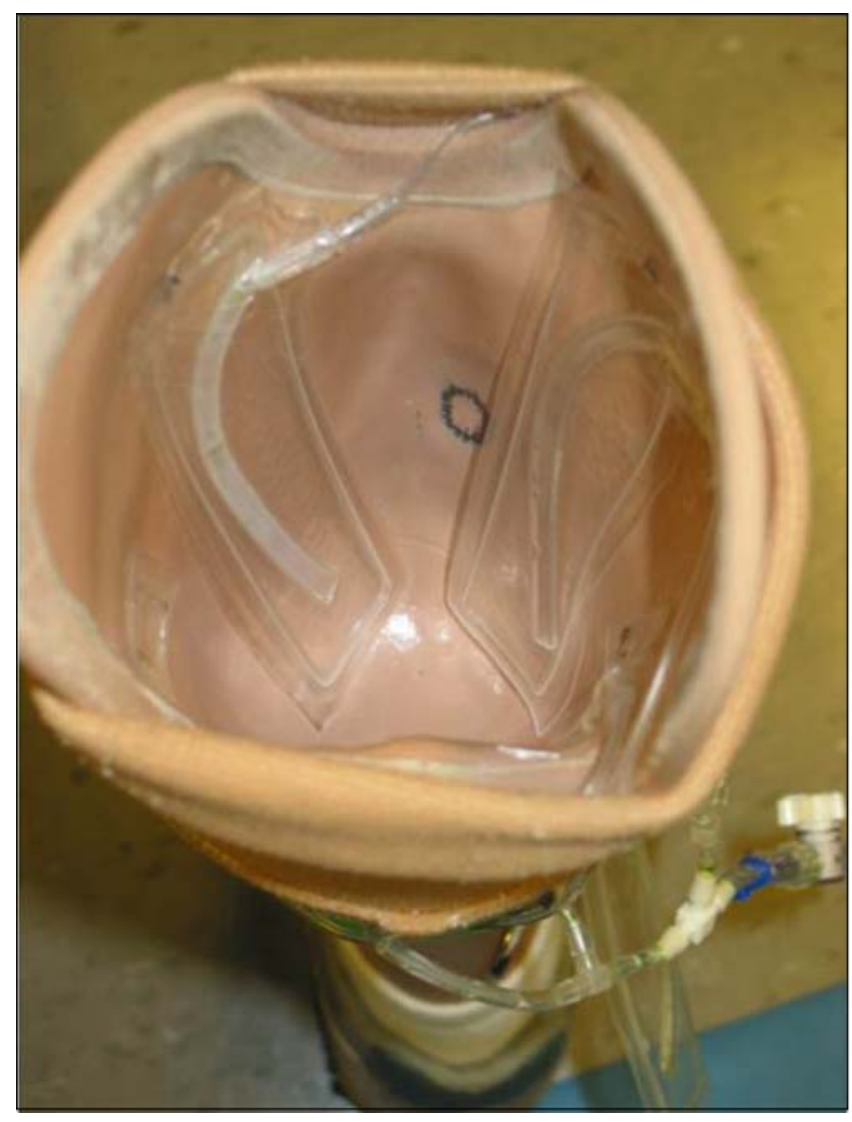

Figure 2.

Instrumented socket. View inside socket with bladders in place.

ment and fluid imbalance detection in hemodialysis patients [14-23]. In four-electrode segmental limb bioimpedance analysis [24], electrical current is applied across two outer electrodes while voltage is sensed between two inner electrodes. The applied current is of low amplitude $(50-700 \mu \mathrm{A})$ and is applied over a frequency range of $5 \mathrm{kHz}$ to $1 \mathrm{MHz}$. The voltage and current signals are demodulated to determine a magnitude and phase difference and then fit to a model to determine extracellular and intracellular impedance [25]. Intracellular and extracellular components can be distinguished because high-frequency current travels well through both intracellular and extracellular biological material while low-frequency current, which does not penetrate cell membranes well, travels primarily through extracellular biological material. Using a limb-segment model [26], we converted impedances to fluid volumes. Only extracellular fluid volume was considered in this analysis because of its likely strong influence on short-term residual-limb 
fluid volume change [2]. In this study, we define "residual-limb fluid volume change" as the extracellular fluid volume change measured using the multifrequency bioimpedance analysis system.

We used a commercial bioimpedance analyzer (Hydra 4200, XiTRON Technologies; San Diego, California) that we modified for testing the residual limbs of people with transtibial limb loss [27]. Briefly, we made custom electrodes using conductive tape (ARcare 8881, Adhesives Research Inc; Glen Rock, Pennsylvania) and an underlying hydrogel (KM10B, Katecho Inc; Des Moines, Iowa). Multistranded silver-plated copper wire (32 AWG, New England Wire Technologies; Lisbon, New Hampshire) was used for the electrode leads. The outside of the conductive tape was covered with Tegaderm transparent film dressing (3M; St. Paul, Minnesota). To prevent air from escaping adjacent to the wires as they passed under the sleeve and out at the thigh, which could reduce suspension, we placed the four wires on a Band-Aid (Johnson \& Johnson; Brunswick, New Jersey) and covered them with Tegaderm. A custom connector with gold-plated pins (WPI Viking, Cooper Interconnect; Maynooth, Ireland) was used to connect the custom electrodes to the instrument, and we replaced the provided connector with a robust cable connector (MS3116F106S, Burndy; Manchester, New Hampshire) to reduce noise from mechanical motion. The smallest volume change that could be resolved was less than 0.1 percent of the residual-limb fluid volume. Data were stored to a personal computer at the sampling rate of the Xitron unit $(1.0 \mathrm{~Hz})$.

Using custom MATLAB version 7.10 (MathWorks; Natick, Massachusetts) code implemented on a separate personal computer, we plotted every other bioimpedance data point in approximately real time ( $3 \mathrm{~s}$ delay). The visual data presentation during the test session helped us to identify any setup problems if they existed.

\section{Protocol}

On a separate day before bioimpedance testing but not more than 12 mo prior, using techniques described in detail previously [28], we tested the subjects for presence of high blood pressure (orthostatic blood pressure $[\mathrm{OBP}]$ ), peripheral arterial disease (ankle-brachial index $[\mathrm{ABI}]$ ), and venous stasis (ambulatory strain-gage plethysmography [ASGP]). We used an electronic blood pressure measurement unit (HEM-775, Omron Healthcare Inc; Lake Forest, Illinois) for OBP testing, a commercial segmental limb pressure measurement system (TD312 cuff inflator, MV10 manifold delector, and SC12 and SC10 cuffs, D. E. Hokanson Inc; Bellevue, Washington) for $\mathrm{ABI}$, and a commercial plethysmography system for ASGP (EC6 plethysmograph, D. E. Hokanson Inc). A practicing endocrinologist interpreted the collected data using standard clinical procedures [29-31].

Subjects were requested not to consume caffeine or alcohol on the morning of the day of bioimpedance testing. After a subject arrived at the laboratory, he or she sat in a chair with the prosthesis donned and the foot supported on the floor for $20 \mathrm{~min}$ while the research practitioner queried the subject about medical history and prosthesis comfort. The entire testing protocol, including audio, was recorded by video camera so it could be reviewed later to capture all verbal information provided by the subject and research practitioner (TASER AXON Flex, Taser International Inc; Scottsdale, Arizona). The subject's mass and height with the prosthesis donned were recorded. The subject then doffed the prosthesis, and the research practitioner inspected the residual limb for signs of injury. If injury was noted, the subject was referred to his or her regular practitioner for treatment and possible socket modification. If injury was not noted, liquid bladders were affixed to the socket with doublestick tape. The bladders were empty at the outset of the trial, but the tubes between the stopcock and bladders were primed with water. Skin sites at which electrodes were to be placed were cleaned with sandpaper (Red Dot Trace Prep 2236, 3M). We applied a very thin layer of ultrasonic coupling gel (Couplant D, GE Panametrics; West Chester, Ohio) between the hydrogel and the subject's skin and then applied the electrodes. The electrodes were applied relative to anatomical landmarks on the residual limb. The proximal voltage-sensing electrode was positioned at the level of the patellar tendon on the lateral posterior limb surface. The distal current-injecting electrode was positioned as far distally as possible but still on the relatively cylindrical portion of the residual limb. The distal voltage-sensing electrode was placed at least $3.0 \mathrm{~cm}$ proximal to the distal current-injecting electrode (Figure 3). The proximal current-injecting electrode was placed on the thigh above the knee but under the elastomeric liner or suspension sleeve. Residual-limb circumference was measured at the levels of the voltagesensing electrodes and the distance between voltagesensing electrode centers was recorded. 


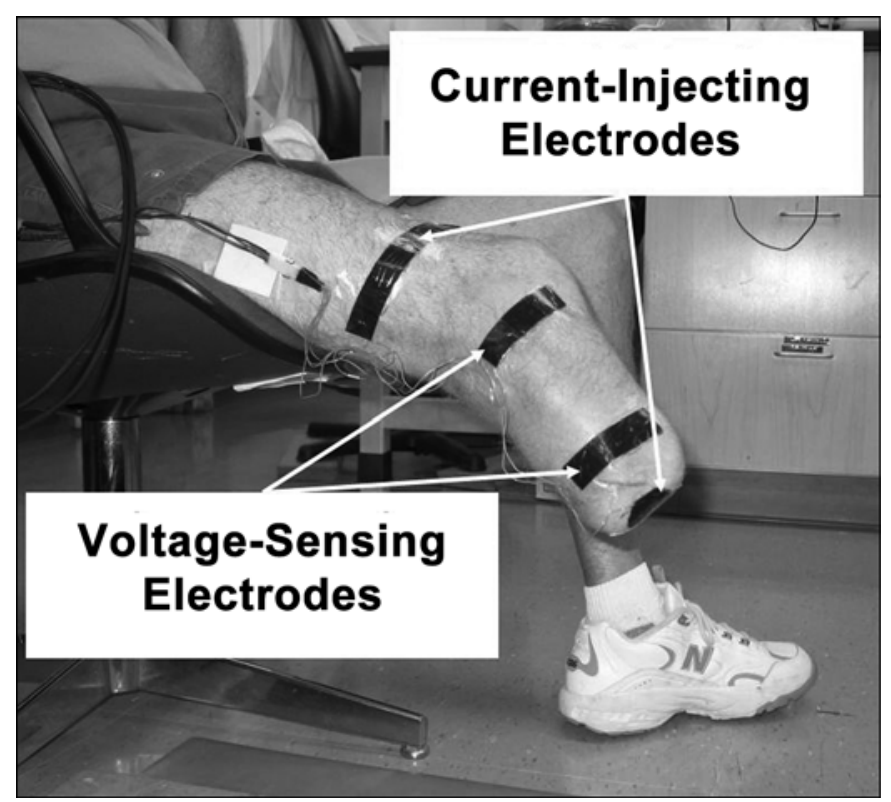

Figure 3.

Residual-limb instrumented with bioimpedance electrodes.

We asked the subject to don his or her prosthesis and then sit for $90 \mathrm{~s}$ with the prosthesis supported by the floor. We took care to ensure the subject maintained a good sitting posture. Too much knee flexion occludes blood flow and too much extension causes a slouching posture. The subject then stood, bearing weight equally for $90 \mathrm{~s}$ on a support platform with an electronic weight scale (349KLX Health-O-Meter, Pelstar LLC; Alsip, Illinois) embedded in the surface. Then, the subject walked on a treadmill (Quinton Clubtrack, Cardiac Science; Bothell, Washington) at a comfortable, self-selected walking speed for $90 \mathrm{~s}$. The subject then stood for $10 \mathrm{~s}$. We considered the point during the $10 \mathrm{~s}$ stand at which the subject achieved equal and stable weight-bearing (typically after about $4 \mathrm{~s}$ ) as the reference residual-limb fluid volume for the session. The basis for using this time point as the reference fluid volume is that in prior investigations, we have found that it takes some subjects one sit/ stand/walk series to adjust to the electrodes and the socket. The cycle of sit/stand/walk, with no liquid in the bladders, was repeated. Then, the subject stood on the electronic scale for $90 \mathrm{~s}$, and we noted the point at which stable equal weight-bearing was achieved. During the subsequent $90 \mathrm{~s}$ sit, liquid was injected into the bladders, either 7 or $5 \mathrm{~mL}$ depending on the practitioner's judgment of how tightly the socket fit. The practitioner used
$5 \mathrm{~mL}$ if the fit was deemed particularly tight and used $7 \mathrm{~mL}$ otherwise. The subject then repeated the sit/stand/ walk series. The subject then sat in the chair, and the bladder liquid that was added before the sit/stand/walk series was removed $(7$ or $5 \mathrm{~mL})$. This process was repeated, adding an additional $7 \mathrm{~mL}$ (or $5 \mathrm{~mL}$ ) in each cycle. Thus, liquid additions were 7, 14, and $21 \mathrm{~mL}$ (or 5, 10 , and $15 \mathrm{~mL}$ ) and so on. We continued adding liquid to the bladders until the subject indicated that the socket was uncomfortable. We also noted the liquid setting the subject considered most comfortable. Two sit/stand/walk series with $0 \mathrm{~mL}$ bladder liquid were performed in succession at the end of the session.

\section{Analysis}

Body mass index (BMI) was calculated as the quotient of mass (kilograms) and the square of height (meters). No correction was made to BMI for the lack of an intact limb.

Total residual-limb volume within the region of interest $\left(\right.$ Volume $\left._{R O I}\right)$, which was between the centers of the voltage-sensing electrodes, was calculated using the circumferential $\left(C_{1}, C_{2}\right)$ and limb length $(L)$ measurements in a truncated cone model, assuming the residual-limb cross-section was circular (Equation (1)):

$$
\text { Volume }_{R O I}(m L)=\frac{L}{12 \pi}\left[C_{1}^{2}+C_{1} C_{2}+C_{2}^{2}\right] \quad \text {. }
$$

To calculate percentage reduction in socket volume for bladder liquid additions, we assumed a total contact socket in the region of interest. We divided the bladder liquid volume by Volume $_{R O I}$ (Equation (2)):

$$
\begin{aligned}
& \% \text { age change in socket volume } \\
& \text { for bladder liquid addition }
\end{aligned}=\frac{\text { bladder liquid addtion }(\mathrm{mL})}{\operatorname{Volume}_{R O I}(\mathrm{~mL})} .
$$

To process the bioimpedance data, we used custom code written in MATLAB that implemented a Cole model algorithm similar to that used by the commercial instrument manufacturer (version 2.2, XiTRON Technologies Inc) [25]. Using limb circumference and voltage electrode separation measurements in an accepted geometric model, we converted the data to extracellular fluid volume [26]. In the analysis, we used only data collected during the brief $10 \mathrm{~s}$ standing intervals, more specifically, 
the point at the onset of equal weight-bearing, after $90 \mathrm{~s}$ walks on the treadmill. All data were expressed as a percentage of fluid volume change relative to the reference volume (ref), which was taken during the first $10 \mathrm{~s}$ standing interval after the first sit/stand/walk series (bladders empty) (Equation (3)):

$$
\% \text { age limb fluid volume change }(t)=\frac{V_{E C F}(t)(m L)}{V_{E C F r e f}(m L)},
$$

where $V_{E C F}=$ extracellular fluid volume.

We calculated the percentage of residual-limb fluid volume change over the session. We also calculated the injection slope, defined as the quotient of percentage of fluid volume change and percentage of change in socket volume for each bladder liquid addition (Equation (4)):

$$
\begin{gathered}
\text { injection slope }= \\
\frac{\text { \%age limb fluid volume change }}{\text { \%age change in socket volume for bladder liquid addition }}
\end{gathered}
$$

Removal slope was calculated (Equation (5)):

$$
\begin{gathered}
\text { removal slope }= \\
\frac{\text { \%age limb fluid volume change }}{\text { \%age change in socket volume for bladder liquid removal }}
\end{gathered}
$$

The limb fluid transport compliance was defined as the difference between the injection slope and removal slope (Equation (6)):

$$
\text { fluid transport compliance }=\text { injection slope }- \text { removal slope. }
$$

We also conducted an exploratory analysis to establish relationships between the calculated measures, characteristics of subject health, and the vascular tests.

\section{RESULTS}

Data were collected from a total of 23 subjects. However, data from four subjects were excluded from analysis: one had metal orthopedic hardware within the thigh of his residuum that distorted the bioimpedance data; one's residual-limb length was too short for the bioimpedance electrodes to be spaced properly; one had too long a spacing between voltage-sensing electrodes, which resulted in impedance measurements outside of the manufacturer's calibration range; and one used an elevated vacuum socket and it was not possible to maintain suspension with the bladders in place. Thus, data from 19 participants are presented next.

The 19 participants averaged $54.2 \pm 10.4 \mathrm{yr}$ (mean \pm standard deviation) in age, $91.4 \pm 19.5 \mathrm{~kg}$ in mass, $180.8 \pm 16.8 \mathrm{~cm}$ in height, and $28.3 \pm 6.7 \mathrm{~kg} / \mathrm{m}^{2}$ in BMI. One subject had bilateral lower-limb amputations, and all other subjects had a unilateral amputation. Time since amputation averaged $14.9 \pm 14.3 \mathrm{yr}$ and mean residuallimb length was $16.4 \pm 3.6 \mathrm{~cm}$. Volume in the region of interest $\left(\right.$ Volume $_{R O I}$ ) averaged $990.4 \pm 290.6 \mathrm{~mL}$. Of the subjects, 12 used an elastomeric liner with locking pin suspension, 3 used a one-way suction valve, and 3 used a suspension sleeve. One used a lanyard suspension. Of the subjects, 7 had peripheral arterial disease, 11 had high blood pressure, and 4 were diabetic. Two subjects had congestive heart failure, and one of those subjects also had kidney failure. Of the subjects, 12 had a BMI greater than $25 \mathrm{~kg} / \mathrm{m}^{2}$, and five of those had a BMI greater than $30 \mathrm{~kg} / \mathrm{m}^{2}$.

Of the 19 subjects in the study, 16 used four bladders and 3 used three bladders. Subjects walked with bladder liquid volumes up to $42.0 \mathrm{~mL}$. The mean maximum bladder liquid volume (i.e., before subjects indicated that the socket was uncomfortable and no more liquid should be added) was $26.3 \pm 8.3 \mathrm{~mL}$. However, the mean maximum bladder liquid volume that subjects preferred was $16.8 \pm$ $8.4 \mathrm{~mL}$, which corresponded with $1.7 \pm 0.8$ percent of the average Volume ${ }_{R O I}$.

There was not a consistent relationship between the percent reduction in socket volume from injecting liquid into the bladders and the resulting percent of residuallimb fluid volume change. The quotient of percentage change in residual-limb fluid volume to percentage reduction in socket volume for bladder liquid additions ranged from -2.5 to 1.0 with a mean of $-0.6 \pm 0.4$ (using 19 means, one for each subject). Thus, on average, for a 1.0 percent decrease in socket size from injecting liquid, we observed a 0.6 percent decrease in residual-limb fluid volume. For liquid removal, the quotient of percentage of change in residual-limb fluid volume to percentage of change in socket volume ranged from -2.0 to 1.5 percent, 
with a mean of $-0.1 \pm 0.4$ percent. Thus, on average, for a 1.0 percent increase in socket size from removing liquid, we observed a 0.1 percent decrease in residual-limb fluid volume. In general, not as much fluid returned to the residual limb after bladder liquid was removed as was displaced out of the residual limb when bladder liquid was added.

The trend of greater residual-limb fluid volume loss upon liquid injection into the bladders than fluid volume gain upon bladder liquid removal was more accentuated earlier in the session than later in the session. There was typically a gradual loss in residual-limb fluid volume over the session (Figure 4(a)). For the 19 subjects, residuallimb fluid volume change from standing after the first walking cycle (defined as the zero reference) to the standing after the last walk at the end of a session ranged from -10.7 to 1.5 percent with a mean of $-3.1 \pm 2.9$ percent. Only one subject showed a change less than -6.0 percent (i.e., $-10.7 \%$ ) and one subject showed a change greater than 1.0 percent (i.e., 1.5\%). Residual-limb fluid volume changes from the beginning to the end of a session for the rest of the subjects were more evenly distributed. Six subjects had changes between -6.0 and -4.0 percent, seven had changes between -4.0 and -1.0 percent, and four had changes between -1.0 and 1.0 percent. The fluid volume changes at the preferred bladder liquid volume, excluding the two subjects for whom a bladder ruptured, averaged $-3.5 \pm 2.7$ percent.

Residual-limb fluid transport compliance, defined as the difference between the injection slope and removal slope (percent/percent) in the fluid volume change versus socket volume change plot (slopes defined in Figure 4(b)) typically reduced with greater liquid volume injected into the bladders (Figure 5(a)-5(s)). A fluid transport compliance value of 0.0 indicates complete residual-limb fluid volume recovery within a bladder injection and removal cycle, while a positive value increasingly further from 0.0 indicates less recovery within an injection and removal cycle. A negative value indicates less fluid volume was lost after bladder liquid was injected than was recovered after bladder liquid was removed (edematous limb).

For the 15 subjects who demonstrated residual-limb fluid volume reduction when liquid was injected into the bladders, we found that the bladder liquid volume for which the socket was most comfortable was not at a residual-limb fluid transport compliance value of 0.0, i.e., complete elastic recovery (Figure 5(a)-5(o)). However, (a)

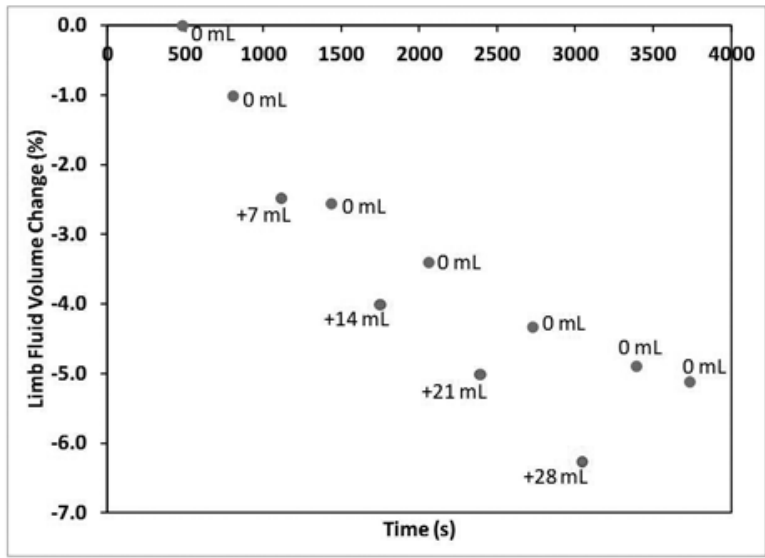

(b)

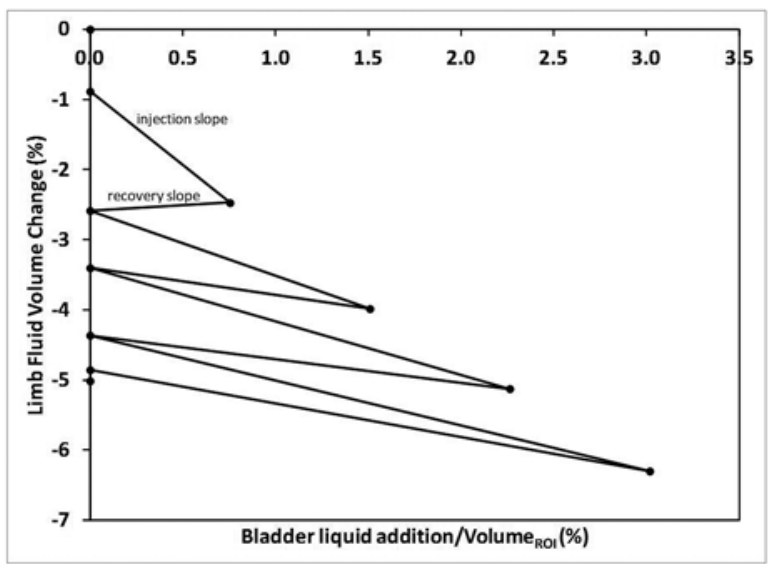

(c)

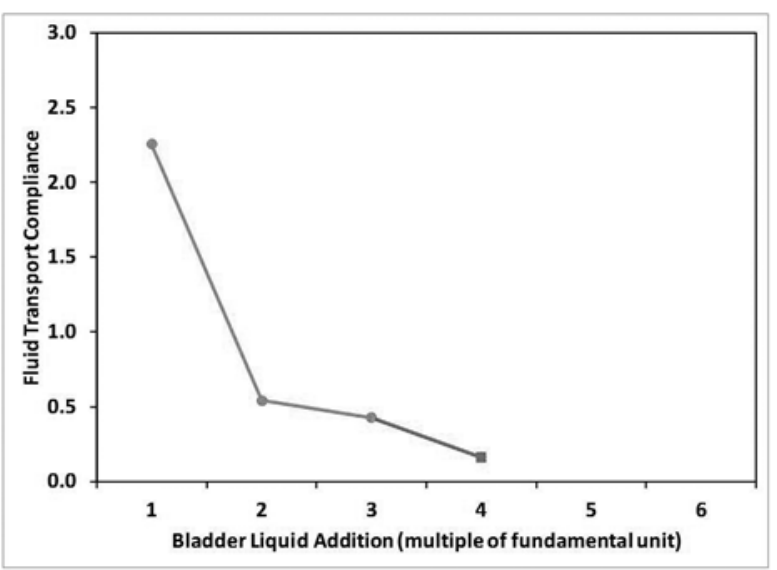

Figure 4.

Example results from subject. Results are typical. (a) Percentage of residual-limb fluid volume change for each bladder liquid addition and removal. Subject showed gradual decrease in fluid volume over session, with increased loss when bladder liquid was added. (b) Percentage of residual-limb fluid volume change versus percentage of socket volume change for each bladder liquid addition and removal. (c) Fluid transport compliance for each bladder liquid addition. $\mathrm{ROI}=$ region of interest. 
more than half of the 15 subjects ( 9 subjects) had reached or were at an inflection point or minimum in their fluid volume fluid transport compliance versus injection cycle plot when the most comfortable bladder liquid volume was reached (Figure 5(a)-5(i)). For five subjects, the most comfortable bladder liquid volume was at a point after the inflection point or minimum (Figure 5(j)5(m)). For two subjects, fluid volume for which the socket was most comfortable could not be determined because the bladder broke during the test (Figure 5(n)5(o)). The residual-limb fluid transport compliance at the most comfortable setting for all subjects except the two for whom the bladders broke ranged from 0.0 to 0.6 with a mean of $0.3 \pm 0.2$. Thus, subjects were still losing some fluid volume at what they considered their most comfortable setting.

Unlike the other 15 subjects, 4 demonstrated residual-limb fluid volume increases upon injection of liquid into the bladders for low bladder liquid volumes at the beginning of the session (Figure 5(p)-5(s)). However, for greater bladder liquid volumes, residual-limb fluid volume decreased upon injection of bladder liquid like it did for the other subjects (Figure 5(a)-5(o)). Only these four subjects demonstrated presence of venous insufficiency in their contralateral limb (ASGP testing). Analysis showed that no other health characteristic we evaluated, including presence of peripheral arterial disease, high blood pressure, obesity (BMI $\geq 30 \mathrm{~kg} / \mathrm{m}^{2}$ ), and smoking, was present in these four subjects and was absent in all other subjects. We did not identify a relationship between health characteristics and the magnitude of residual-limb fluid volume change.

\section{DISCUSSION}

Change in residual-limb volume is a source of discomfort for people using prosthetic limbs. The purpose of this research was to gain insight into how adding and removing liquid from bladders positioned on the inside socket wall affected residual-limb fluid volume.

We considered how the instrumentation and study design may have affected study results. Since the changes in residual-limb fluid volume we measured were much greater than the resolution of the bioimpedance instrument $(<0.1 \%$ limb fluid volume), it is unlikely that instrument error affected our findings. We recognize that placing the bladders at locations different from those we selected might affect the results. We placed the bladders anteriorly at locations recommended by Simbex, manufacturer of the automated Active Contact System, and posteriorly at locations expected not to occlude major blood vessels. Different locations might affect flow through major vessels, e.g., the posterior tibial vasculature, which might affect residual-limb fluid volume. A sensitivity analysis of fluid volume change to bladder location needs to be conducted. The ordering of liquid volumes added and removed might also have affected results. For example, reversing the ordering of liquid volume additions from largest to smallest might have avoided the gradual fluid volume loss over the test session we observed with the conducted protocol and instead induced a more distinct inflection point in the compliance plot (Figure 1(c)).

The socket volume changes we induced in the present study for the preferred bladder liquid volume averaged $1.7 \pm 0.8$ percent, less than that induced during weight-bearing with addition of a new 1-ply polyester blend sock (mean of 2.2\%) [12]. However, in the present study, the socket reduction was highly localized to the anterior and posterior proximal regions. Thus, the liquid additions induced a local residual-limb fluid volume change (mean for preferred bladder volume was $-3.5 \% \pm$ $2.7 \%$ ), much higher than were measured on the group of 28 subjects who added a 1-ply polyester blend sock to their socket (mean of $-0.9 \%$ ) [28].

We did not implement a control system in this investigation to adjust bladder volume according to a threshold pressure measured within the bladders, like the Simbex Active Contact System, but instead made bladder volume the controlled variable. We did this to scientifically test the effect of bladder liquid volume on the independent variable of residual-limb fluid volume. Because of this methodology, we cannot draw conclusions about the clinical performance of the Simbex Active Contact System or another socket-adjusting product with a built-in controller. Based on experience in this and related studies using bioimpedance analysis on subjects with amputation, we would expect the ordering and timing of liquid additions and removals to strongly influence residual-limb fluid volume change. Possibly, a built-in controller would be effective if it was programmed to avoid overfilling the bladders during activity and emptying the bladders during inactivity, reducing fluid volume loss and facilitating residual-limb fluid volume recovery, respectively. In other words, the ordering of liquid additions and their durations 

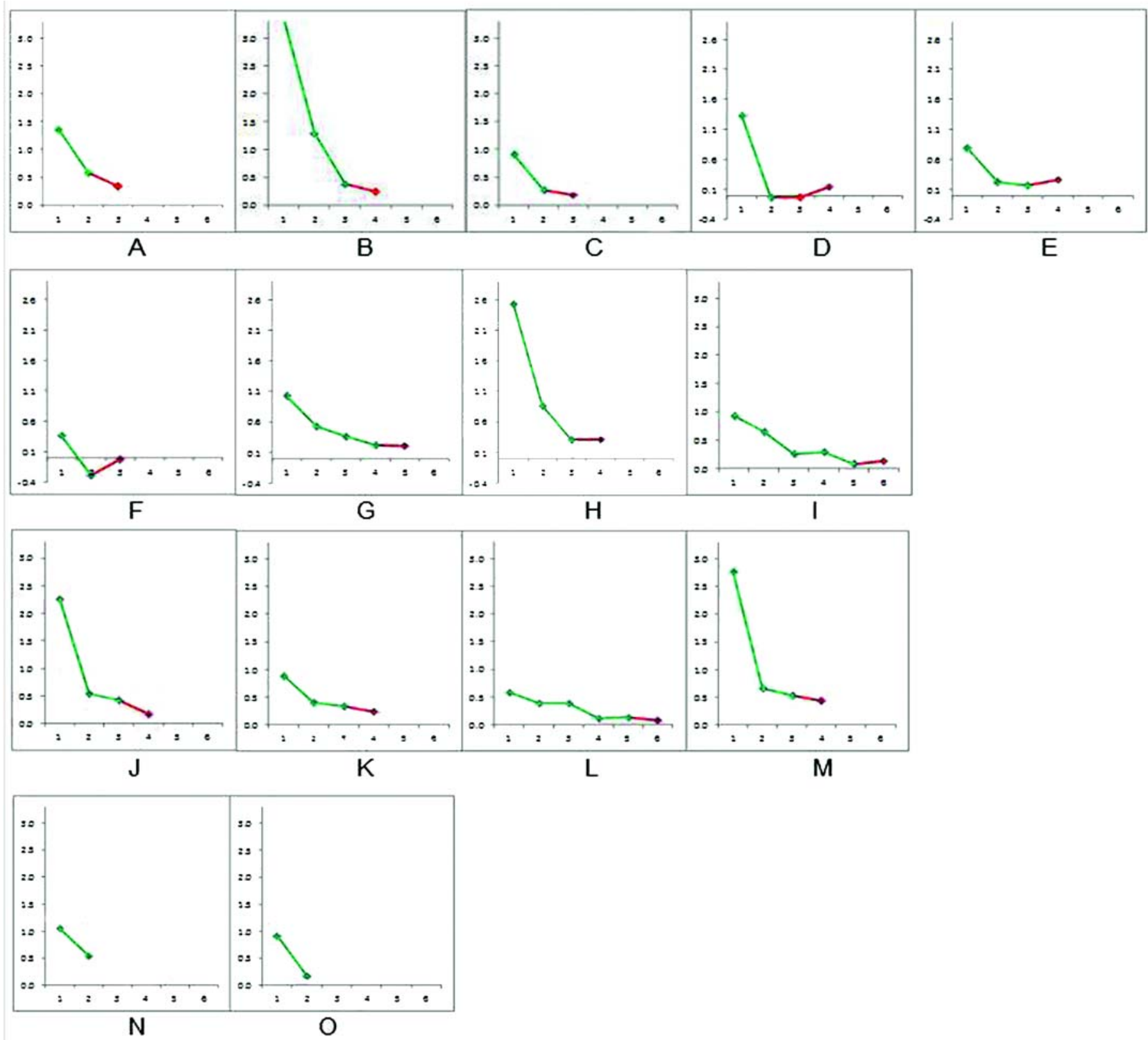

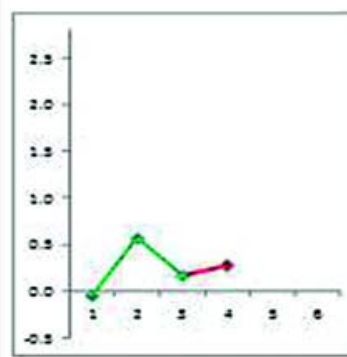

P

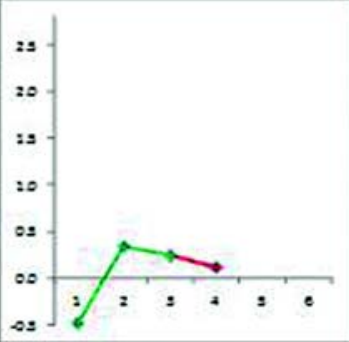

Q

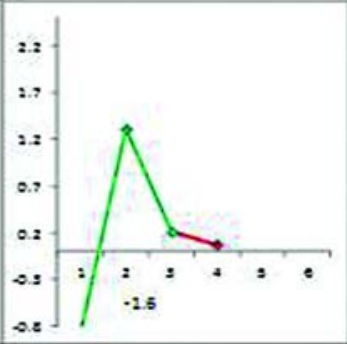

$\mathrm{R}$

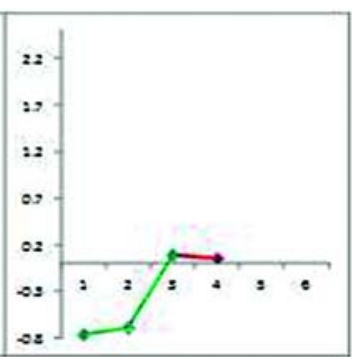

$\mathrm{S}$

Figure 5.

Residual-limb fluid transport compliance. Results for all 19 subjects are shown. (a)-(i) Subjects were at primary inflection point or minimum in their transport compliance curve when most comfortable bladder liquid setting was reached, indicated by transition to red line. (j)-(m) Subjects were beyond primary inflection point when most comfortable bladder liquid setting was reached. (n)-(o) Subjects for whom bladder broke during testing. (p)-(s) Subjects showed initial increase in residual-limb fluid transport compliance, followed by decrease. 
accomplished with an automated system might not induce as high fluid volume losses as we measured using the protocol in the present investigation. A worthwhile next study would be to test sockets equipped with built-in controllers and compare fluid volume changes with those induced using the present protocol.

The range of bladder liquid volume each subject tolerated varied considerably across the 15 subjects, from as low as $15 \mathrm{~mL}$ to as high as $42 \mathrm{~mL}$ (not including the two subjects for whom a bladder broke). We expect that this result reflects differences in initial socket fit as well as variability in residual-limb mechanical characteristics. Subject residual limbs varied in stiffness, size, and load tolerance. Similarly, there was not a consistent relationship between the percentage of socket volume reduction induced by injecting liquid into the bladders and the resulting percentage of residual-limb fluid volume change. It ranged from -2.5 to 1.1 percent. Again, we expect that this result reflects variability in initial socket fit as well as differences in subject residual-limb qualities. The relevance of this result is that it is not possible to state clinical guidelines of how much residual-limb fluid loss will occur for each milliliter of bladder liquid injected. Possibly in the future, analytical models could be generated to relate subject and socket qualities, limb and socket shape difference, limb mechanical properties, and bladder volumes to residual-limb fluid volume change. Then, potential changes in residual-limb fluid volume could be predicted for the individual patient for each milliliter of bladder liquid addition.

The results from the present study suggest that fluid volume driven out of the residual limb as a result of adding liquid to the bladders was not easily recovered when the bladder liquid was removed. This result points to the history dependence of residual-limb fluid transport. This behavior is an important consideration for engineers designing adjustable-socket technologies and for practitioners fitting these systems to patients. It is not reasonable to assume that one bladder volume will induce only one residual-limb fluid volume, even within the same day. Designers need to create controller technology to carefully adjust socket volume so that excessive residuallimb fluid volume losses are not induced. Results from the present study indicate that a means for the practitioner to control the maximum bladder liquid level the patient can inject may be needed. We believe that residual-limb fluid volume recovery strategies, not just strategies that tighten the socket on the limb, are relevant to consider. If the residual limb reduces in volume when socket size is reduced and is not allowed to recover, through either passive or active actuation, then the residual limb could become dehydrated and possibly atrophy over the long term.

The result that fluid volume driven out of the residual limb when bladder liquid was added was not recovered upon bladder liquid removal is also relevant to clinical fitting procedures. During prosthetic-fitting sessions, the practitioner must be mindful of previous socket configurations affecting the patient's present residual-limb volume. It is not reasonable to assume that the patient will return to the same residual-limb size when the socket is returned to the same size. This result is similar to findings from a prior study where we added and then removed socks on subjects [28]. We suggest a physiological explanation for residual-limb fluid loss over time: while a person wears a prosthesis, unless the socket is very loose, interface pressures induce interstitial fluid pressures that put residual-limb fluid transport out of balance. Elevated interstitial fluid pressures cause greater interstitial to venous transport than arterial to interstitial transport. The result is a loss of residual-limb fluid volume over time. Thus, just wearing the socket, even without activity, may cause a loss in residual-limb fluid volume.

We defined compliance to provide a measure of how well the residual limb bounced back and recovered fluid volume after liquid added to bladders was removed. Because compliance values were slightly greater than 0.0 at high bladder volumes in the present study, we conclude that the residual-limb response at high bladder volumes was close to, but not completely, elastic. There was still fluid volume loss. While the physiological bioprocesses that caused this loss are unclear, we can provide a physical description that may be useful toward clinical understanding. Practitioners can think of the residual limb as a sponge; fluid was quickly expelled when the prosthesis user was active and the residual limb was squeezed within a tight socket (much liquid in bladders), but that fluid was not easily recovered when the tight socket was released. We expected the transition point, the transition from a loose socket to a stable and comfortable socket, to be at or near the intersection of the steep slope and shallow slope portions of the compliance-bladder volume curve (Figure 1(c)) because we would not expect the increased interface pressures at higher bladder volumes to be worth a marginal increase in stability. In general, 
this expectation held in the data collected here (Figure 5), with most subjects preferring a liquid volume at the transition from a steep to shallow slope in the compliance curve or at the next-highest liquid volume level. We are unsure why subjects with shallow slopes in their initial compliance curves tended to prefer very high bladder liquid volumes.

Some subjects showed a relatively narrow range in their fluid transport compliance plot (e.g., Figure 5(i)), while others demonstrated a larger range (e.g., Figure 5(h)). This difference in results between subjects may reflect, in part, socket fit at the outset of testing. We would expect subjects with tightly fitting sockets at the outset to experience less fluid transport compliance because there is less interstitial fluid and blood available to transport out of the residual limb. However, this expectation is conjecture at this point and would need to be scientifically validated.

Of the 19 subjects in the present study, 4 demonstrated an increase in residual-limb fluid volume with liquid addition to the bladders. This edematous behavior was observed early in the test session when small bladder liquid volumes were injected. We observed a related trend, an increase in residual-limb fluid volume with sock addition, in 6 of 28 subjects in a previous investigation when a sock was added to the residual limb [28]. Reports in the literature show that nondisabled subjects tend to increase in limb fluid volume over the day, a result thought to reflect the effects of gravity pulling fluid distally into the limbs [32-34]. Thus, for subjects in the present study who increased in residual-limb fluid volume with liquid addition to the bladders, we expect that the interface pressures from walking with a prosthetic socket did not offset the influence of gravity pulling fluid into the limbs. Other than presence of venous insufficiency, we did not note any other physiologic or health differences in these four subjects compared with other subjects. Venous insufficiency may have curtailed venous transport relative to arterial transport until bladder fluid volumes were increased to the point that interstitial pressures were elevated enough to induce greater interstitialto-venous transport. This point is reflected as a positive to negative slope change in the fluid transport compliance plot.

Findings from the present study for most of the 15 subjects who did not demonstrate edematous behavior support our expectation that there was fluid within the residual limb that was easily expelled from the first blad- der fill and remove cycle (Figure 1(a)). Subsequently, there was stiffer resistance to expelling fluid. Because this initial fluid did not easily return, we might interpret it to be stagnant "nonessential" fluid. Note that no subject preferred the initial setting but instead preferred smaller socket sizes where there was more resistance to residuallimb fluid loss. Thus, our data, in general, support the concepts illustrated in Figure 1. Since we performed two "zero" bladder volume cycles initially at the outset of the session and referenced fluid volume change to the end of the first sit/stand/walk series, we do not believe that this initial loss represents the departure of quick-moving fluid that entered the residual limb while the subject sat to have the electrodes placed. It instead reflected the effects of bladder liquid addition and removal.

An emerging challenge in prosthetics technology is to design effective controllers to operate adjustablesocket technologies. We believe that the extremes of the fluid transport compliance curve represent the extremes of fit. When fluid transport compliance is high (low bladder liquid volumes in Figure 1(c)), the mechanical coupling between the socket and residual limb may be weak and the person using the prosthesis is at risk of instability and falling. At high bladder liquid volumes, subjects did not experience zero fluid transport compliance; thus, it is reasonable to conclude that there is little advantage, once stability is accomplished, to further elevate bladder liquid levels. Over-filling the bladders beyond the point necessary to accomplish stability may put the prosthesis user at risk of excessive fluid volume loss, tissue dehydration, and soft-tissue injury. While the present study demonstrated that most subjects achieved residual-limb fluid volume change versus socket-size change plots similar to that shown in Figure 1(c), the subject selected an optimal setting typically at or after this inflection point. An interesting next step would be to test whether subjects continue to select these settings over longer test periods, e.g., 1 day, or whether they prefer lower settings nearer the inflection point in the fluid transport compliance plot. An additional intriguing question is whether the transition point shifts over time.

A needed area of research is to determine to what degree interface pressures need to be decreased so as to induce residual-limb fluid volume recovery. This insight would not only help users stabilize residual-limb fluid volume but would also facilitate the design of adjustablesocket control systems to accomplish recovery, relieving the user of the burden. For subjects with poor fluid balance 
between arterial-to-interstitial and interstitial-to-venous transport, we suggest that either prosthesis doffing or negative pressure (i.e., vacuum-assist) is needed to accomplish residual-limb fluid volume recovery.

An engineering challenge is a versatile bladder sizing and positioning technology that is easy to implement in prosthetic sockets. Computer-aided design/computeraided modeling socket manufacturing technology offers a platform for custom bladder design and positioning for the individual patient that may facilitate application of bladder liquid technology to prosthetics.

\section{CONCLUSIONS}

The maximum bladder liquid volume subjects tolerated ranged to $42.0 \mathrm{~mL}$. However, the preferred bladder liquid volume was $16.8 \pm 8.4 \mathrm{~mL}$, corresponding with $1.7 \pm 0.8$ percent of the average socket volume in the region of interest. The point the prosthesis user preferred bladder liquid volume during ambulation was not at a point where fluid out equaled fluid in for an injection and removal cycle. There was not a consistent relationship between the percent of reduction in socket volume from injecting liquid into the bladders and the resulting percent of residual-limb fluid volume change. This result held across subjects and for different bladder liquid additions on the same subject. Residual-limb fluid volume driven out of the residual limb when bladder liquid was added was typically not recovered upon subsequent bladder liquid removal. Of the 19 subjects, 15 experienced a gradual residual-limb fluid volume loss over the test session. Four subjects experienced fluid volume gains when bladder liquid was added. These were the only subjects with the presence of venous insufficiency. Care should be taken when implementing adjustable-socket technologies in people with limb amputation. Reducing socket volume may accentuate residual-limb fluid volume loss. Recovery strategies, e.g., enlarging the socket during rest periods, may facilitate fluid volume recovery and reduce daily fluid volume loss.

\section{ACKNOWLEDGMENTS}

\section{Author Contributions:}

Study concept and design: J. E. Sanders, K. J. Allyn.

Acquisition of data: D. S. Harrison, T. R. Myers, K. J. Allyn. Analysis and interpretation of data: J. C. Cagle, J. E. Sanders.
Drafting of manuscript: J. E. Sanders, J. C. Cagle.

Critical revision of manuscript for important intellectual content:

K. J. Allyn, J. C. Cagle.

Statistical analysis: J. C. Cagle.

Obtained funding: J. E. Sanders.

Financial Disclosures: The authors have declared that no competing interests exist.

Funding/Support: This material was based on work supported by the Orthotic and Prosthetic Education and Research Foundation and the

National Institutes of Health (American Rehabilitation and Recovery Act, grant R01HD60585).

Additional Contributions: We appreciate assistance from D. E. Hokanson Inc for RF-welding the bladders. Mr. Myers is now with Newberg Roadhouse LLC, Seattle, Washington.

Institutional Review: All study procedures were reviewed by an internal human subjects review board, and approval was received before any study procedures were initiated.

Participant Follow-up: The authors do not plan to inform participants of the publication of this study. However, participants have been encouraged to check the study Web site for updated publications.

\section{REFERENCES}

1. Sanders JE, Fatone S. Residual limb volume change: systematic review of measurement and management. J Rehabil Res Dev. 2011;48(8):949-86. [PMID:22068373] http://dx.doi.org/10.1682/JRRD.2010.09.0189

2. Zachariah SG, Saxena R, Fergason JR, Sanders JE. Shape and volume change in the transtibial residuum over the short term: preliminary investigation of six subjects. J Rehabil Res Dev. 2004;41(5):683-94. [PMID:15558398] http://dx.doi.org/10.1682/JRRD.2003.10.0153

3. Allyn KJ, Cagle JC, Phillips RH, Harrison DS, Hartley TL, Sanders JE. Maintaining limb volume: Effects of doffing between periods of activity. Proceedings of the 38th American Academy of Orthotists \& Prosthetists Academy Annual Meeting and Scientific Symposium; 2012 Mar 2124; Atlanta (GA).

4. Caspers CA, inventor. Hypobarically-controlled artificial limb for amputees. United States patent US 5,549,709 A. 1996 Aug 27.

5. Caldwell RJ. Troubleshooting sub-atmospheric systems. The Academy Today. 2012;8(1):A-13.

6. Ogawa A, Obinata G, Hase K, Dutta A, Nakagawa M. Design of lower limb prosthesis with contact pressure adjustment by MR fluid. Conf Proc IEEE Eng Med Biol Soc. 2008;2008:330-33. [PMID:19162660] http://dx.doi.org/10.1109/IEMBS.2008.4649157

7. Sanders JE, Cassisi DV. Mechanical performance of inflatable inserts used in limb prosthetics. J Rehabil Res Dev. 2001;38(4):365-74. [PMID:11563488]

8. Robert DC, Mayor MB, Nelson DF, Bradley CS, Blanchard MW, inventors. Simbex LLC, assignee. Dynamic variable 
geometry fitting system for use with a body appliance. United States patent US 6,585,774 B2. 2003 Jul 1.

9. Greenwald RM, Dean RC, Board WJ. Volume management: Smart Variable Geometry Socket (SVGS) technology for lower-limb prostheses. J Prosthet Orthot. 2003; 15(3):107-12.

10. Phillips VL, inventor. Socket insert having a bladder system. United States patent US 7,655,049 B2. 2010 Feb 2.

11. Ezenwa B, inventor. Wisys Technology Foundation, assignee. Prosthetic socket with real-time dynamic control of pressure ports. United States patent US 7,670,386 B2. 2010 Mar 2.

12. Sanders JE, Cagle JC, Harrison DS, Karchin A. Amputee socks: how does sock ply relate to sock thickness? Prosthet Orthot Int. 2012;36(1):77-86. [PMID:22228614] http://dx.doi.org/10.1177/0309364611431290

13. Sanders JE, Severance MR, Allyn KJ. Computer-socket manufacturing error: how much before it is clinically apparent? J Rehabil Res Dev. 2012;49(4):567-82. [PMID:22773260] http://dx.doi.org/10.1682/JRRD.2011.05.0097

14. Van Loan MD, Withers P, Matthie J, Mayclin PL. Use of bioimpedance spectroscopy to determine extracellular fluid, intracellular fluid, total body water, and fat-free mass. Basic Life Sci. 1993;60:67-70. [PMID:8110166]

15. Organ LW, Bradham GB, Gore DT, Lozier SL. Segmental bioelectrical impedance analysis: theory and application of a new technique. J Appl Physiol. 1994;77(1):98-112. [PMID:7961281]

16. Fuller NJ, Hardingham CR, Graves M, Screaton N, Dixon AK, Ward LC, Elia M. Predicting composition of leg sections with anthropometry and bioelectrical impedance analysis, using magnetic resonance imaging as reference. Clin Sci (Lond). 1999;96(6):647-57. [PMID:10334971] http://dx.doi.org/10.1042/CS19980376

17. Salinari S, Bertuzzi A, Mingrone G, Capristo E, Scarfone A, Greco AV, Heymsfield SB. Bioimpedance analysis: a useful technique for assessing appendicular lean soft tissue mass and distribution. J Appl Physiol. 2003;94(4):1552-56. [PMID:12626475]

18. Dittmar M. Reliability and variability of bioimpedance measures in normal adults: effects of age, gender, and body mass. Am J Phys Anthropol. 2003;122(4):361-70.

[PMID:14614757]

http://dx.doi.org/10.1002/ajpa.10301

19. Andreoli A, Melchiorri G, De Lorenzo A, Caruso I, Sinibaldi Salimei P, Guerrisi M. Bioelectrical impedance measures in different position and vs dual-energy X-ray absorptiometry (DXA). J Sports Med Phys Fitness. 2002; 42(2):186-89. [PMID:12032414]

20. de Vries PM, Meijer JH, Vlaanderen K, Visser V, Oe PL, Donker AJ, Schneider H. Measurement of transcellular fluid shift during haemodialysis. Part 2. In vitro and clinical evaluation. Med Biol Eng Comput. 1989;27(2):152-58. [PMID:2601434] http://dx.doi.org/10.1007/BF02446224

21. Zhu F, Schneditz D, Wang E, Martin K, Morris AT, Levin NW. Validation of changes in extracellular volume measured during hemodialysis using a segmental bioimpedance technique. ASAIO J. 1998;44(5):M541-45.

[PMID:9804490]

http://dx.doi.org/10.1097/00002480-199809000-00045

22. Shulman T, Heidenheim AP, Kianfar C, Shulman SM, Lindsay RM. Preserving central blood volume: changes in body fluid compartments during hemodialysis. ASAIO J. 2001;47(6):615-18. [PMID:11730198] http://dx.doi.org/10.1097/00002480-200111000-00009

23. Donadio C, Consani C, Ardini M, Bernabini G, Caprio F, Grassi G, Lucchesi A, Nerucci B. Estimate of body water compartments and of body composition in maintenance hemodialysis patients: comparison of single and multifrequency bioimpedance analysis. J Ren Nutr. 2005;15(3): 332-44. [PMID:16007563]

http://dx.doi.org/10.1016/j.jrn.2005.04.001

24. Grimnes S, Martinsen OG. Bioimpedance and bioelectricity basics. 2nd ed. London (England): Academic Press; 2008.

25. De Lorenzo A, Andreoli A, Matthie J, Withers P. Predicting body cell mass with bioimpedance by using theoretical methods: a technological review. J Appl Physiol. 1997;82(5): 1542-58. [PMID:9134904]

26. Fenech M, Jaffrin MY. Extracellular and intracellular volume variations during postural change measured by segmental and wrist-ankle bioimpedance spectroscopy. IEEE Trans Biomed Eng. 2004;51(1):166-75. [PMID:14723506] http://dx.doi.org/10.1109/TBME.2003.820338

27. Sanders JE, Allyn KJ, Harrison DS, Myers TR, Ciol MA, Tsai EC. Preliminary investigation of residual-limb fluid volume changes within one day. J Rehabil Res Dev. 2012; 49(10):1467-78. [PMID:23516051] http://dx.doi.org/10.1682/JRRD.2011.12.0236

28. Sanders JE, Harrison DS, Allyn KJ, Myers TR, Ciol MA, Tsai EC. How do sock ply changes affect residual-limb fluid volume in people with transtibial amputation? J Rehabil Res Dev. 2012;49(2):241-56. [PMID:22773526] http://dx.doi.org/10.1682/JRRD.2011.02.0022

29. Adragao T, Pires A, Branco P, Castro R, Oliveira A, Nogueira C, Bordalo J, Curto JD, Prata MM. Ankle- brachial index, vascular calcifications and mortality in dialysis patients. Nephrol Dial Transplant. 2012;27(1):318-25. [PMID:21551082] http://dx.doi.org/10.1093/ndt/gfr233

30. Potier L, Abi Khalil C, Mohammedi K, Roussel R. Use and utility of ankle brachial index in patients with diabetes. Eur 
J Vasc Endovasc Surg. 2011;41(1):110-16. [PMID:21095144]

http://dx.doi.org/10.1016/j.ejvs.2010.09.020

31. Nicolaides AN; Cardiovascular Disease Educational and Research Trust; European Society of Vascular Surgery; The International Angiology Scientific Activity Congress Organization; International Union of Angiology; Union Internationale de Phlebologie at the Abbaye des Vaux de Cernay. Investigation of chronic venous insufficiency: A consensus statement (France, March 5-9, 1997). Circulation. 2000; 102(20):E126-63. [PMID:11076834] http://dx.doi.org/10.1161/01.CIR.102.20.e126

32. Ludbrook J, Loughlin J. Regulation of volume in postarteriolar vessels of the lower limb. Am Heart J. 1964;67:493-507. [PMID:14138811] http://dx.doi.org/10.1016/0002-8703(64)90096-1

33. Stick C, Stöfen P, Witzleb E. On physiological edema in man's lower extremity. Eur J Appl Physiol Occup Physiol. 1985;54(4):442-49. [PMID:4065133] http://dx.doi.org/10.1007/BF02337192

34. Stick C, Jaeger H, Witzleb E. Measurements of volume changes and venous pressure in the human lower leg during walking and running. J Appl Physiol. 1992;72(6):2063-68. [PMID:1629057]

Submitted for publication June 29, 2012. Accepted in revised form January 8, 2013.

This article and any supplementary material should be cited as follows:

Sanders JE, Cagle JC, Harrison DS, Myers TR, Allyn KJ. How does adding and removing liquid from socket bladders affect residual-limb fluid volume? J Rehabil Res Dev. 2013;50(6):845-60.

http://dx.doi.org/10.1682/JRRD.2012.06.0121

ResearcherID/ORCID: Joan E. Sanders, PhD: E-8204-2011

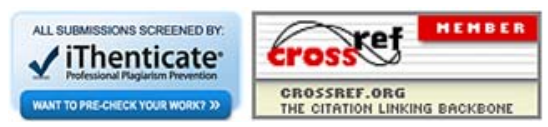


